\title{
Metagabros de la isla Gran Roque (Venezuela). Geoquímica y petrografía
}

\author{
Luz Mary Toro-Toro*, Mario Moreno-Sánchez, Arley de Jesús Gómez-Cruz, Juliana Giraldo-García \\ Departamento de Ciencias Geológicas, Facultad de Ciencias Exactas y Naturales, Universidad de Caldas, Manizales, Colombia
}

\begin{abstract}
Resumen
La isla Gran Roque, situada al norte de Venezuela, es una de las pocas islas del sur del Caribe en donde afloran rocas de corteza oceánica. Estas rocas corresponden a metagabros de grano fino a grueso, con textura relicto subofítica definida por cristales de plagioclasa rodeados por minerales ferromagnesianos.

La asociación de actinolita, clorita, plagioclasa y cuarzo indica que la roca alcanzó un grado metamórfico bajo en facies de esquisto verde. La composición geoquímica de las rocas de la isla Gran Roque es coherente con la clasificación petrográfica. Estas rocas muestran afinidad toleítica y patrones planos de tierras raras, lo que indica que provienen de magmas generados en dorsales oceánicas de tipo MORB-N.
\end{abstract}

Palabras clave: Gran Roque, meta-gabros, MORB-N, Venezuela.

\begin{abstract}
Metagabbros of Gran Roque Island (Venezuela). Geochemistry and petrography

The Gran Roque Island, located in northern Venezuela, is part of the southern Caribbean islands where the basic basement is outcropping. This basement is composed of fine-grained metagabbros, with subophitic relict texture defined by the larger plagioclase laths that enclose the ferromagnesian minerals.

The mineral assemblage of chlorite-actinolite-plagioclase-quartz indicates that the rock reached a low metamorphic grade green schist facies. The chemistry of the Gran Roque island metaggabros supports the petrographic identification and characterization. These rocks exhibit tholeiitic affinity and REE flat patterns, which could be correlated to magmas generated at oceanic ridges of N-MORB type.
\end{abstract}

Key words: Gran Roque, metagabbros, N-MORB, Venezuela.

\section{Introducción}

El grosor de la corteza oceánica "normal" rara vez supera los 10 kilómetros; sin embargo, se calcula que en las áreas de emisión de basaltos de meseta (plateau o, en sentido más amplio, "grandes provincias ígneas" o LIP, del inglés large igneous provinces), el espesor de la corteza puede alcanzar entre 15 y 30 kilómetros (Sinton, 1996; Sinton, et al., 1998). La mayor parte de la placa del Caribe se atribuye a la actividad de un punto caliente, el que se presume estuvo situado en el océano Pacífico durante el Cretácico tardío y que produjo una fuerte actividad ígnea intrusiva y extrusiva que engrosó la corteza (Donnelly, et al., 1973; Edgar, et al., 1971). La placa del Caribe es un segmento de litosfera terrestre conformado principalmente por una corteza oceánica de rocas básicas cubierto por el mar Caribe, con una extensión de 3,2 millones de kilómetros y un promedio de profundidad de 2.500 metros. Debido a su gran espesor, la placa del Caribe pudo haberse emplazado por obducción sobre el margen occidental del norte de Sudamérica, formando una serie de terrenos producto de la acreción en los Andes de Colombia y Ecuador (Kerr, et al., 1996a; Saunders, et al., 1996).

El espesor de la placa del Caribe no es uniforme: varía desde los $8 \mathrm{~km}$ en la corteza oceánica normal hasta los $20 \mathrm{~km}$ en la corteza engrosada o plateau. Aunque la corteza engrosada es una característica dominante en la placa, también es común encontrar corteza normal del tipo extensional, como en la cresta de Beata y el escarpe de Hess (Sinton, et al., 1998). El acceso directo al basamento de la placa del Caribe ha estado limitado a las perforaciones hechas durante el Deep Sea Drilling Project (DSDP) y el Ocean Drilling Program (ODP), por medio de los cuales se ha reconocido la estructura y composición de los gabros y basaltos de esta

\section{*Correspondencia:}

Luz Mary Toro-Toro, luz.toro@ucaldas.edu.co

Recibido: 7 de mayo de 2014

Aceptado: 15 de septiembre de 2014 
placa (Donnelly, et al., 1973). Son evidentes las dificultades para acceder al basamento de la placa del Caribe in situ, sin embargo, la exposición del basamento en "ventanas tectónicas" (Sinton, et al., 1998) en los márgenes de la placa es una oportunidad para estudiar la estructura y la composición de estas rocas.

El margen sur de la placa del Caribe, que limita con el norte de Sudamérica, es una zona tectónicamente difusa y de gran complejidad estructural. El límite puede trazarse dentro de una amplia faja constituida por una serie de fallas de rumbo y cabalgamientos que afectan el norte de Colombia, las islas de Barbados, Trinidad, Tobago, Aruba, el archipiélago de los Roques y la costa norte de Venezuela. Además, la placa del Caribe es geológicamente importante, ya que es una de las grandes provincias ígneas (LIP) del mundo, cuyo origen se sitúa en el océano Pacífico durante el Cretácico tardío (Duncan \& Hargraves, 1984).

El archipiélago de los Roques, cerca de la costa norte de Venezuela, es uno de los pocos lugares al sur de la placa del Caribe en los que esta expone su suelo, lo cual es geológicamente importante, ya que su basamento puede arrojar luz sobre la posible homogeneidad geoquímica de dicha placa.

Según Schubert \& Moticska (1972), las rocas del basamento que afloran en la isla Gran Roque (Venezuela), situada al sur de la placa del Caribe, están enmarcadas dentro del denominado "complejo metaígneo de Gran Roque". La isla tiene una superficie de $1,7 \mathrm{~km}^{2}$, constituida en su mayor parte por depósitos recientes; en el sector norte se exponen cerca de $0,6 \mathrm{~km}^{2}$ de basamento constituido principalmente por metadiabasas y metagabros (Gonzáles de Juana, et al., 1980; Ostos, 1990; Urbani, 2005); según Schubert \& Moticska (1972), las rocas de la isla están afectadas tanto por metamorfismo de contacto como por metamorfismo regional bajo en la zona de clorita. Las islas de Gran Roque y La Orchila, $60 \mathrm{~km}$ al este, se encuentran intruidas por rocas ácidas, entre las que se cuentan cuerpos graníticos, diques aplíticos, y diques pegmatíticos; sin embargo, en $\mathrm{La}$ Orchila se expone un zócalo más antiguo constituido por esquistos, filitas y neises (Schubert \& Moticska, 1972). En la isla de Gran Roque las rocas más antiguas corresponden a anfibolitas y gabros metamorfoseados con una edad de 130 \pm 14 millones de años (Ma), e intruídas por rocas félsicas de una edad de $65 \pm 3,6 \mathrm{Ma}$, edades calculadas con el método K/Ar (Santamaría \& Schubert, 1974). Según el análisis geoquímico presentado por Ostos (1990), los metabasaltos y metadiabasas de la isla son de naturaleza toleítica, y su ambiente geológico es de basaltos de dorsal. En cuanto a las rocas graníticas, Ostos (1990) concuerda con Santamaría y Schubert (1974) en que estas se originaron en un arco de islas volcánico.
El objeto de este trabajo es aportar nuevos datos petrográficos y geoquímicos de las rocas de la placa del Caribe que afloran en la isla Gran Roque (Venezuela). Los resultados obtenidos en este trabajo servirán en parte como base de comparacion con bloques o terrenos que se sospecha podrían ser fragmentados de la placa del Caribe original.

\section{Localización}

El área de estudio (isla Gran Roque) se localiza en el mar Caribe, en el extremo noroeste del archipiélago de Los Roques, el cual se encuentra dentro de la cadena de las islas Aruba - Los Testigos, al norte de las costas venezolanas (Figura 1). Las coordenadas geográficas que limitan la zona de estudio son $11^{\circ} 56^{\prime} 35^{\prime \prime}$ y $11^{\circ} 57^{\prime} 32^{\prime \prime}$ latitud norte, $66^{\circ}$ $41^{\prime} 49^{\prime \prime}$ y $66^{\circ} 39^{\prime} 47^{\prime \prime}$ longitud oeste. La isla Gran Roque tiene forma de cuña alargada en dirección noroeste, tiene una longitud de $3 \mathrm{~km}$ y un ancho máximo de $1 \mathrm{~km}$ (Figura 1), su área es de aproximadamente de $1,7 \mathrm{~km}^{2}$ (Schubert \& Moticska, 1972). Dicha isla está a unos $168 \mathrm{~km}$ al norte de La Guaira, puerto de Caracas. A la zona de estudio se accede por vía marítima o aérea.

\section{Metodología}

Se tomaron muestras para análisis petrográficos y geoquímicos de roca total, estos últimos elaborados por los Laboratorios AcmeLabs (Acme Analytical Laboratories) en Vancouver $\mathrm{BC}$, Canadá, mediante el método de espectrometría de masas con fuente de plasma de acoplamiento inductivo (Inductively Coupled Plasma - Mass Spectrometry, ICP MS). La precisión que se logra por este procedimiento es de \pm 2 y \pm 5 para una concentración analítica entre 50 y 5 ppm, respectivamente. El análisis modal se llevó a cabo utilizando un conteo de 500 puntos.

\section{Marco geológico regional}

La región Caribe es muy compleja desde el punto de vista tectónico, ya que es el resultado de la interacción de la placa del Caribe con las placas de Nazca, Cocos, Norteamérica y Sudamérica. Los límites actuales de la placa del Caribe son los siguientes.

Al este, con la zona de subducción de las placas de Norteamérica y Sudamérica por debajo de la placa del Caribe, dando lugar al arco volcánico de las Antillas Menores.

El límite occidental es la zona de subducción entre las placas de Cocos y Nazca, que subducen bajo la placa del Caribe, dando lugar al arco volcánico centroamericano.

Al norte, el límite se encuentra dominado por el desplazamiento transcurrente con la placa de Norteamérica, desde el centro de Guatemala hasta el norte de las Antillas Menores, que se manifiesta mediante extensos sistemas de fallas de desgarre. 

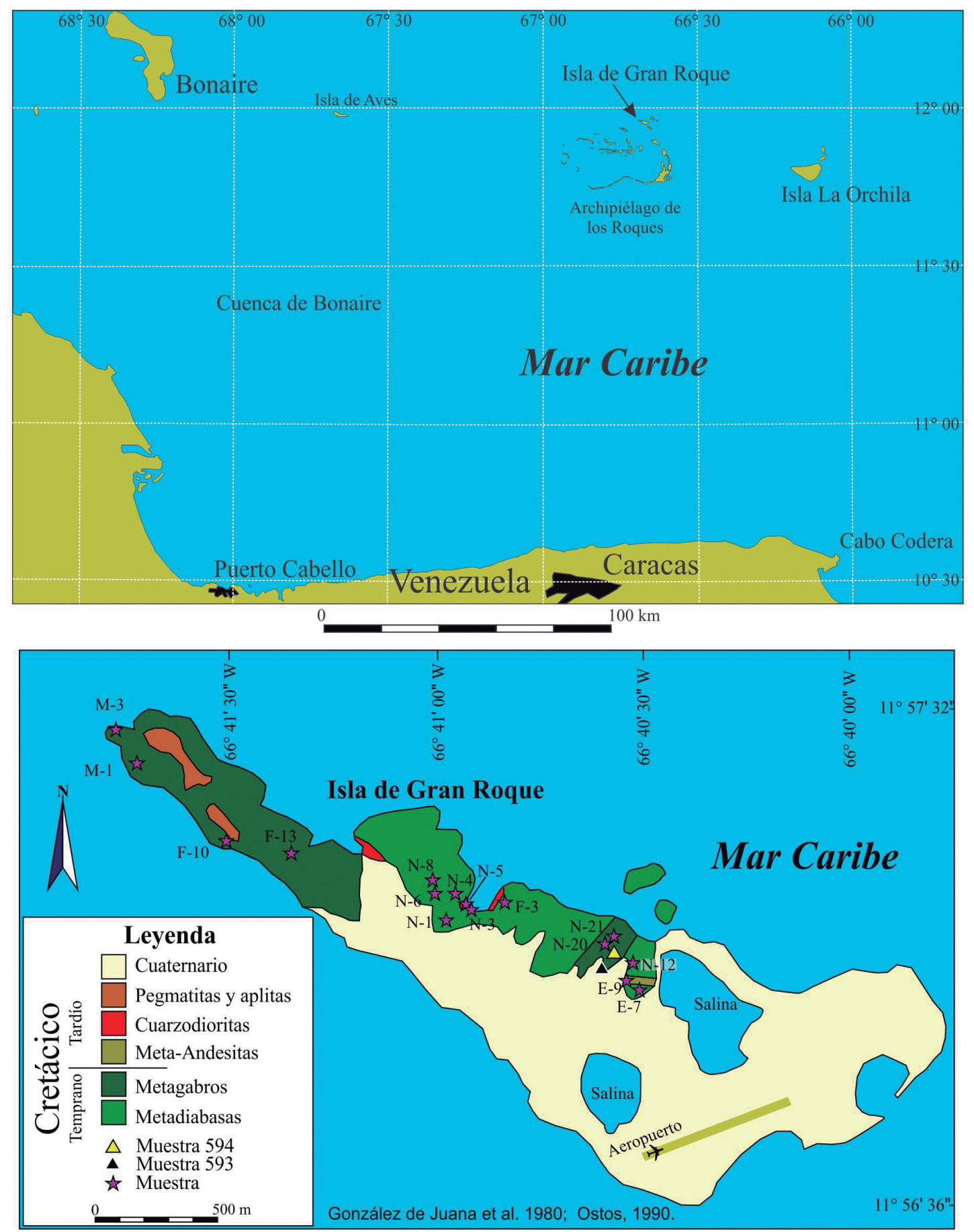

Figura 1. Mapa Geológico y de localización de La Isla Gran Roque, Archipiélago Los Roques (Venezuela). Las estrellas indican los sitios de muestreo de Ostos (1990) y los triángulos de este trabajo.

Por último, el límite sur, ha actuado de manera transcurrente con un sentido dextro durante la mayor parte de la historia geológica y en la actualidad funciona como una zona de subducción en la que la placa del Caribe se consume por debajo de la de Sudamérica (Draper \& Gutiérrez, 1997).
La reconstrucción de la historia evolutiva se dificulta debido a la ausencia de patrones magnéticos, lo que sugiere centros de expansión en la mayor parte de la placa (James, 2007). El único centro de expansión activo desde el Oligoceno en la placa del Caribe es la cuenca de Cayman. La placa del 
Caribe no es fisiográficamente uniforme y puede dividirse en tres grandes cuencas separadas por accidentes submarinos: la cuenca de Granada, separada de la de Venezuela por el cresta de Aves, y la cuenca de Colombia, separada de la de Venezuela por la cresta de Beata. La característica más notable de la placa del Caribe es que presenta una corteza con espesor mayor al promedio de la corteza oceánica y menor que el espesor de la corteza continental, aspecto reconocido inicialmente por Officer, et al., (1957), e interpretado como corteza oceánica con espesor anómalo (Edgar, et al., 1973). La corteza de la placa del Caribe, sin embargo, no es homogénea, pues al occidente de la cresta de Beata su espesor es de $20 \mathrm{~km}$ y hacia el sureste de la cuenca de Venezuela es muy delgado, entre 2 y 5 km (Diebold \& Driscoll, 1999). El espesor en las cuencas de Granada y Colombia oscila entre los 10 y 22 km (Case, et al., 1990).

La isla Gran Roque es una pequeña extensión de tierra al norte del archipiélago con apenas 1,7 km cuadrados de extensión, de los cuales solo $0,6 \mathrm{~km}$ representan afloramientos de basamento. La parte restante de la isla corresponde a depósitos marinos cuaternarios. En los sectores de los cerros occidental y oriental afloran metagabros, denominados “gabros hornbléndicos” (Aguerrevere \& López, 1938). La metadiabasa, referida por Aguerrevere \& López (1938) como diabasa de grano fino, es la roca que constituye el cerro central y la parte oeste del oriental. En los tres cerros se presentan intrusiones de cuarzo-diorita que forman pequeños cuerpos irregulares y diques. Por último, todas estas rocas fueron intruidas por diques y venas de composición aplítica. Las rocas presentan un intenso diaclasamiento, y es muy común encontrar material fosfático de color verde pistacho, amorfo, que se encuentra en venas a través de la roca caja. Esta fosfatización es producto de la alteración de la roca caja producida por soluciones de ácido fosfórico provenientes del guano y de las lluvias.

\section{Petrografía}

Los metagabros son rocas de grano fino a grueso, de color verde oscuro a negro, que conservan sus rasgos primarios, entre ellos su textura relicto subofítica. El componente mineralógico primario es la plagioclasa y los intersticios se encuentran ocupados por piroxenos (Figura 2). El piroxeno se presenta en cristales subhedrales-anhedrales de un tamaño que varía entre 0,1 y 0,7 mm; es de color verde pálido con pleocroísmo y su composición es augítica. La composición de la plagioclasa en cristales euhedrales con macla tipo albita corresponde a (An ${ }_{60-70}$ ), obtenida mediante el método de Michel-Levy. En cuanto a la mineralogía secundaria, esta corresponde a anfiboles, clorita, epidota, carbonatos, cuarzo y esfena, minerales estos que se presentan en reemplazo de los piroxenos (Figura 2a). Los anfiboles secundarios se presentan en cristales con hábito acicular, de color verde claro a amarillo
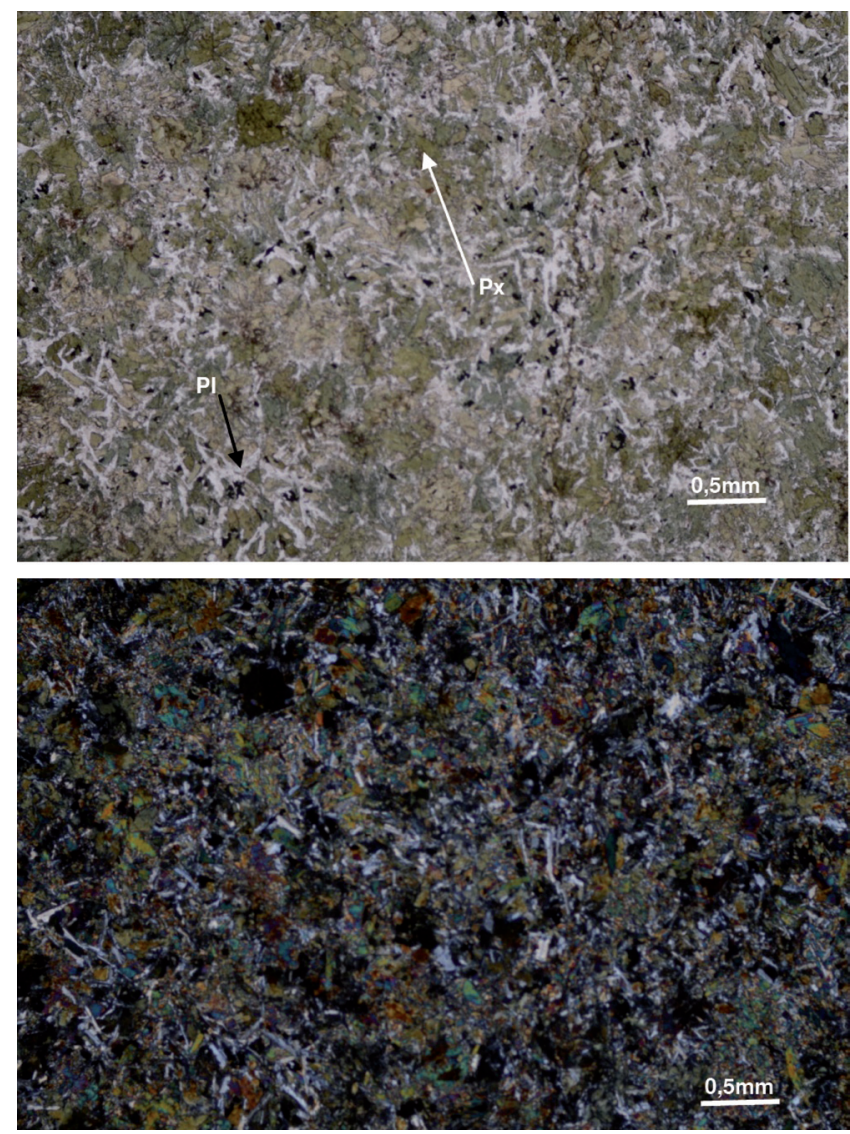

Figura 2. Aspecto petrográfico de los metagabros con textura relicto subofítica definida por cristales de plagioclasa (Pl) euhedral y los intersticios ocupados por piroxenos (Px).

pálido, con tamaños menores de $0,2 \mathrm{~mm}$ con pleocroísmo, identificados como actinolitas. La epidota se presenta en cristales prismáticos cortos o granulares en contacto con la actinolita. La clorita en masas micáceas es de tamaño muy fino, de color verde, con leve pleocroísmo, y color de interferencia café del primer orden. Tanto el cuarzo como los carbonatos ocupan los espacios.

La paragénesis metamórfica está representada por actinolita, plagioclasa, clorita, cuarzo, carbonatos y epidota, que representan condiciones que alcanzaron el grado bajo en facies esquisto verde. No es fácil determinar la composición de la plagioclasa producto del proceso metamórfico debido al tamaño de los cristales, que es menor de $0,1 \mathrm{~mm}$.

\section{Geoquímica}

En la Tabla 1 se presentan los resultados de los análisis químicos realizados; los diagramas fueron elaborados en el programa Geochemical Data Toolkit (GCDKit 3.0). Según la petrografia, las rocas estudiadas de la isla Gran Roque han sido afectadas por procesos metamórficos e hidrotermales, 

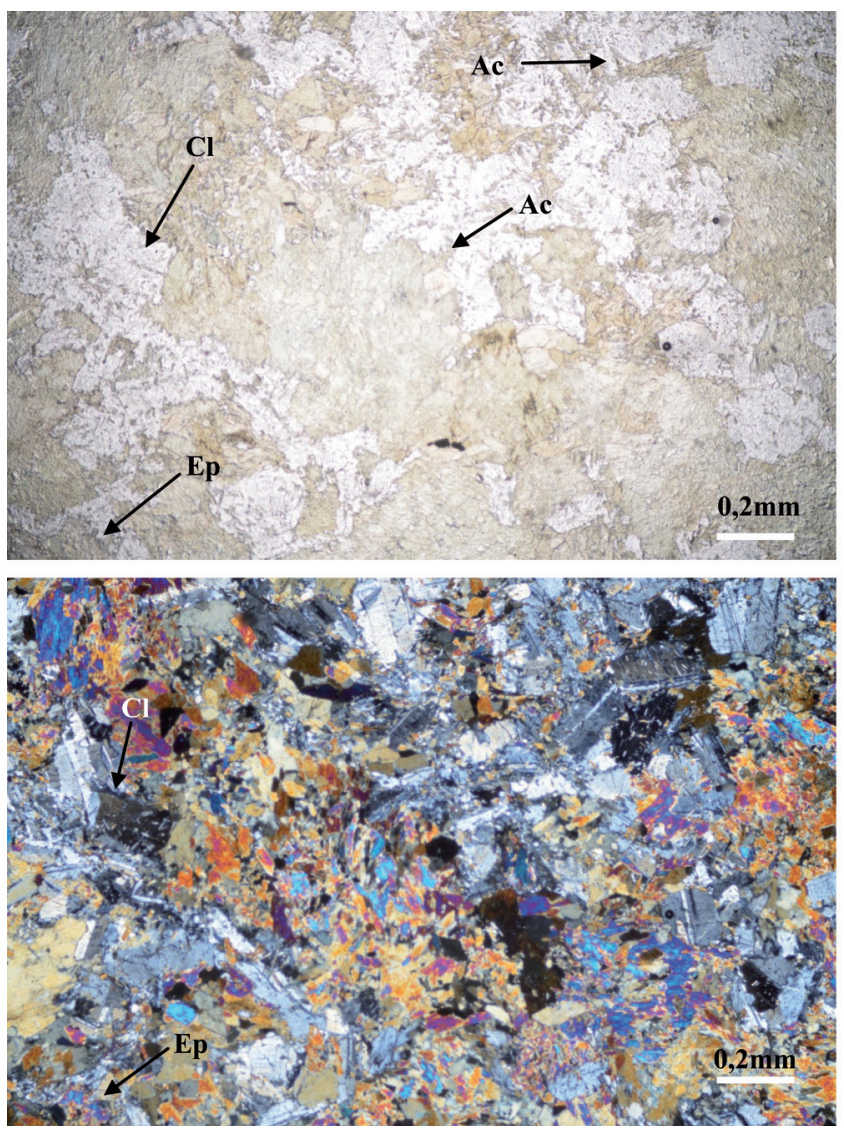

Figura 2a. La mineralogía secundaria que se desarrolla a partir de piroxenos que se transforman anfiboles aciculares de actinolita (Ac) y los cristales plagioclasa en epidota (Ep), carbonatos $(\mathrm{Ca})$ y clorita $(\mathrm{Cl})$.

que probablemente produjeron movilidad de elementos, lo cual modifica la composición química original de la roca; de ahí que se deben emplear diagramas en los que se usen, preferentemente, elementos inmóviles (Pearce, 1996). Las discrepancias observadas entre los datos geoquímicos de este trabajo y los datos de Ostos (1990), pueden deberse a las diferencias analíticas de los laboratorios, por tanto los datos de este autor no fueron usados en las gráficas presentadas.

En el diagrama de clasificación de rocas volcánicas de Winchester \& Floyd (1976), en el cual se proyectan las rocas plutónicas, se grafica así: $\mathrm{Nb} / \mathrm{Y} V s$. $\mathrm{SiO}$, En la Figura 3a se observa que las muestras analizadas corresponden a rocas basálticas subalcalinas de afinidad toleítica. En el diagrama ternario AFM (Irvine \& Baragar, 1971), se evidencian claramente los bajos niveles de álcalis y el carácter toleítico de las rocas gabroícas muestreadas en la isla Gran Roque (Figura 3b).

En el diagrama de tierras raras (rare earth element, REE), normalizadas con respecto a la condrita según el procedimiento de Nakamura (1974), las rocas muestran un muy
Tabla 1. Análisis químicos de elementos mayores y trazas incluyendo las tierras raras. $\mathrm{El} \mathrm{Fe}_{2} \mathrm{O}_{3}$ representa el $\mathrm{Fe}$ total

\begin{tabular}{|c|c|c|}
\hline Muestras & M 593 & M 594 \\
\hline \multicolumn{3}{|c|}{ Elementos Mayores (\% peso) } \\
\hline $\mathrm{SiO}_{2}$ & 48,82 & 48,32 \\
\hline $\mathrm{Al}_{2} \mathrm{O}_{3}$ & 14,47 & 14,21 \\
\hline $\mathrm{Fe}_{2} \mathrm{O}_{3}$ & 10,98 & 7,70 \\
\hline $\mathrm{MgO}$ & 9,16 & 12,23 \\
\hline $\mathrm{CaO}$ & 11,83 & 13,12 \\
\hline $\mathrm{Na}_{2} \mathrm{O}$ & 4,77 & 1,37 \\
\hline $\mathrm{K}_{2} \mathrm{O}$ & 0,08 & 0,11 \\
\hline $\mathrm{TiO}_{2}$ & 0,97 & 0,32 \\
\hline $\mathrm{P}_{2} \mathrm{O}_{5}$ & 0,21 & 0,32 \\
\hline $\mathrm{Cr}_{2} \mathrm{O}_{3}$ & 0,058 & 0,18 \\
\hline LOI & 1,2 & 1,50 \\
\hline Total & 99,76 & 99,70 \\
\hline \multicolumn{3}{|c|}{ Elementos trazas (ppm) } \\
\hline $\mathrm{Ba}$ & 16 & 191 \\
\hline $\mathrm{Ga}$ & 14,4 & 10,4 \\
\hline Hf & 1,4 & 0,4 \\
\hline $\mathrm{Nb}$ & 3,2 & 0,9 \\
\hline $\mathrm{Rb}$ & 1 & 2,1 \\
\hline $\mathrm{Sn}$ & $<1$ & 1 \\
\hline $\mathrm{Sr}$ & 129,2 & 130,1 \\
\hline $\mathrm{Ta}$ & 0,3 & 0,1 \\
\hline Th & $<0,2$ & $<0,2$ \\
\hline $\mathrm{U}$ & $<0,1$ & $<0,1$ \\
\hline V & 337 & 213 \\
\hline $\mathrm{Zr}$ & 46,8 & 18,3 \\
\hline Y & 18,6 & 9,2 \\
\hline $\mathrm{La}$ & 2,7 & 1 \\
\hline $\mathrm{Ce}$ & 7,5 & 2,9 \\
\hline $\operatorname{Pr}$ & 1,13 & 0,46 \\
\hline $\mathrm{Nd}$ & 5,3 & 2,5 \\
\hline $\mathrm{Sm}$ & 1,84 & 0,91 \\
\hline $\mathrm{Eu}$ & 0,69 & 0,34 \\
\hline $\mathrm{Gd}$ & 2,65 & 1,18 \\
\hline $\mathrm{Tb}$ & 0,5 & 0,25 \\
\hline Dy & 3,33 & 1,61 \\
\hline Но & 0,71 & 0,32 \\
\hline $\mathrm{Er}$ & 2 & 1,07 \\
\hline $\mathrm{Tm}$ & 0,29 & 0,15 \\
\hline $\mathrm{Yb}$ & 1,81 & 0,9 \\
\hline $\mathrm{Lu}$ & 0,3 & 0,16 \\
\hline
\end{tabular}


a.

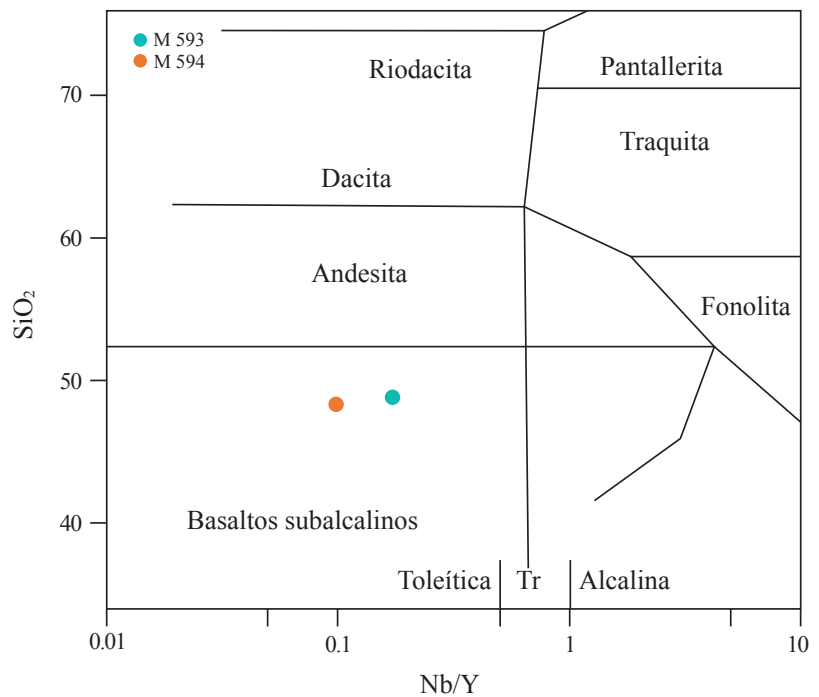

b.

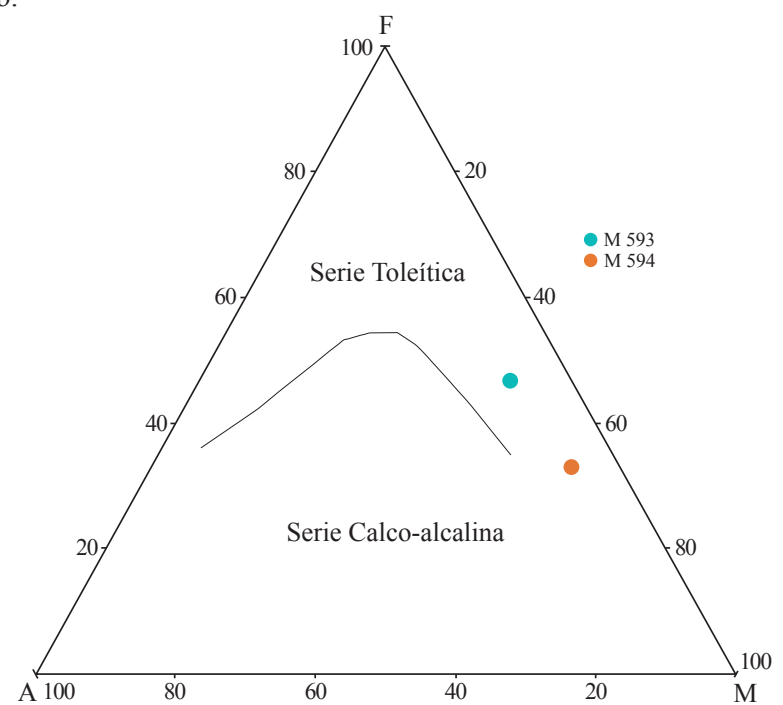

Figura 3. a. Diagrama de clasificación de Winchester \& Floyd (1976). Los límites toleítico (Tol), transicional (Tr) y alcalino (Alc) corresponden a Pearce (1982). b. Diagrama AFM para dividir rocas subalcalinas, en Serie Toleítica y Serie Calco-alcalina, propuesto por Irvine \& Baragar (1971).

a.

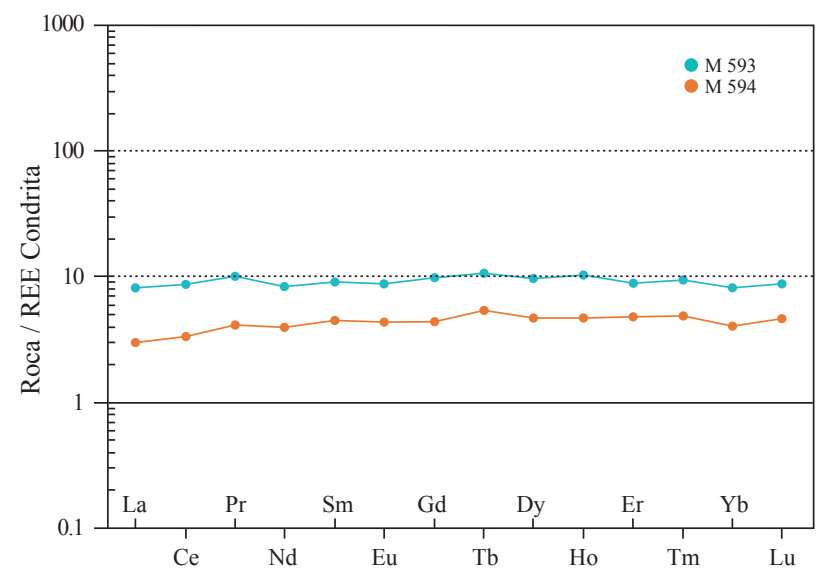

b.

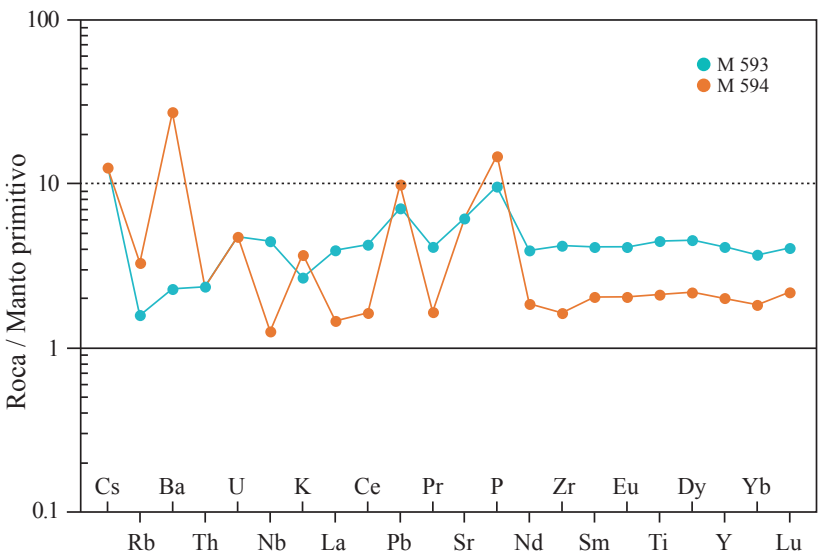

Figura 4. a. Diagrama de REE normalizadas con respecto a la condrita (Nakamura, 1974). b. Diagramas multielementos normalizados con respecto a manto primitivo (Sun \& McDonough, 1989)

leve empobrecimiento en elementos de las tierras raras livianas (light rare earth element, LREE) con respecto a elementos de tierras raras pesadas (heavy rare earth element, HREE), y se observa que los patrones de normalización son relativamente planos y homogéneos. En este diagrama es evidente un enriquecimiento con respecto al valor de la condrita del orden de 10 y 3 veces, comportamiento típico de rocas generadas en una dorsal meso-oceánica tipo MORB-N (Figura 4a). La relación $(\mathrm{La} / \mathrm{Lu})_{\mathrm{N}}$ para las muestras M 593 y M 594 tiene valores de 0,92 y 0,64 y la relación $(\mathrm{La} / \mathrm{Sm})_{\mathrm{N}}$ presenta valores de 0,90 y 0,68 , respectivamente, lo que significa que estas muestras tienen similitud con rocas de dorsal oceánica tipo MORB-N, en la que ambas relaciones son menores de 1 (Wilson, 1989).

En el diagrama de multielementos propuesto por Sun \& McDonough (1989), en el cual se normaliza con respecto a la composición del manto primitivo, estas rocas revelan patrones similares a MORB y en él se observa un acentuado enriquecimiento en $\mathrm{Ba}$, de hasta 30 veces, en la muestra $\mathrm{M}$ 594. Además, se observan anomalías positivas en $\mathrm{Cs}, \mathrm{Pb}$ y $\mathrm{Sr}$ 
en ambas muestras. Esta dispersión en las concentraciones puede responder al metamorfismo, por medio del cual los elementos más móviles vuelven a movilizarse durante estos procesos (Figura $4 b$ ).

Para determinar en qué tipo de MORB se formaron las rocas, se utilizó el diagrama ternario de discriminación $\mathrm{Zr} / 4-2 \mathrm{Nb}$ - Y para rocas basálticas propuesto por Meschede (1986), en el cual las muestras se ubican en el campo de rocas generadas en una dorsal meso-oceánica y cuyas fuentes provienen del segmento MORB-N en el manto superior (Figura 5a). Este resultado se confirma usando el diagrama $\mathrm{Nb} / \mathrm{Y} V s . \mathrm{TiO}_{2} / \mathrm{Y}$ (Pearce, 2007) (Figura 5b).

\section{Discusión}

Las rocas metamórficas que afloran en la parte central de la isla Gran Roque al norte de Venezuela, corresponden a metagabros y se vieron afectadas por un metamorfismo de bajo grado que alcanzó la facies esquisto verde, conservando su textura original subofitica.

En cuanto a la geoquímica, las rocas tienen una tendencia toleítica, con indicios de formación en un ambiente de dorsal meso-oceánica normal (MORB-N). La isla Gran Roque es una de las pocas exposiciones in situ de la corteza oceánica del Caribe, de ahí que los resultados de este trabajo aportan información geoquímica básica para la comparación con terrenos alóctonos que se han interpretado como fragmentos de esta placa. Al comparar las concentraciones de los elementos traza de las muestras analizadas con los valores planteados para las rocas generadas en ambiente MORB y de plateau, establecidos en el Basaltic Volcanism Study Proyect de 1981 (Wilson, 1989, Nivia, 1987, y Kerr, et al., 1997), se puede ver que los valores de las rocas estudiadas caen en el

a.

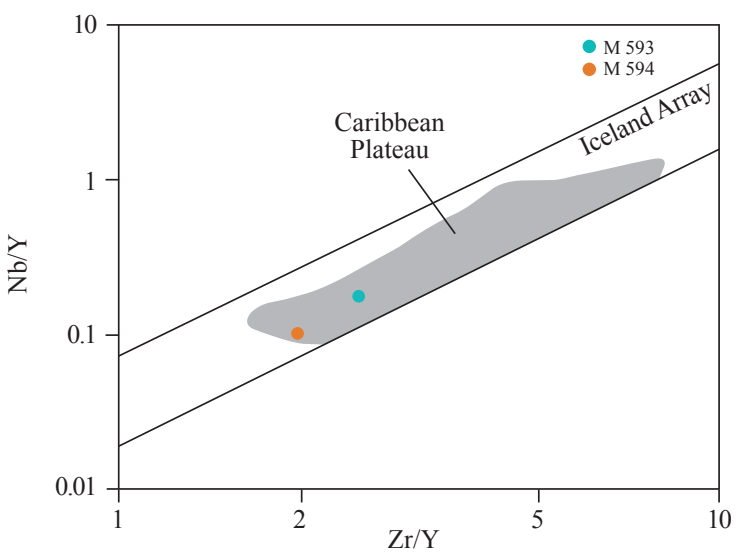

campo de dorsal meso-oceánica (MORB). De igual manera, muestran similitudes con rocas generadas en el Plateau del Caribe, lo que concuerda con la propuesta de Kerr, et al., (1996b), Fitton, et al., (1997), y Thompson, et al., (2003).

Según el modelo planteado por Pindell \& Kennan (2001), la placa del Caribe nace en el Cretácico temprano sobre un punto caliente (¿Galápagos?), situado en el océano Pacífico. Durante el Cenozoico la placa se desplazó hacia el este en el espacio dejado por Norte y Sudamérica (James, 2006). Según el modelo de evolución propuesto por Pindell (1994), las rocas de la Cordillera Occidental de Colombia se originaron en el margen de la placa del Caribe, por lo que Kerr, et al., (2000) las denominan Plateau oceánico caribe-colombiano (Caribbean-Colombian Oceanic Plateau, CCOP). La subducción de la placa Protocaribe (corteza oceánica normal) bajo el margen de la placa del Caribe (constituida en su mayor parte por rocas de plateau) da origen al arco de las Antillas (Kerr, et al., 2000; Moreno-Sánchez \& PardoTrujillo, 2003; Pindell, 1994), lo que explica la presencia de granitoides calcoalcalinos que intruyen las rocas básicas en el margen sur de la placa del Caribe y el occidente colombiano. La isla Gran Roque, junto con las islas de Aruba, Curazao, Aves, Bonaire, La Orchila y la Blanquilla, son, al parecer, los remanentes del desplazamiento de la placa y el arco del Caribe hacia el este durante el Cenozoico, como lo sugiere en estos lugares la presencia de un basamento oceánico Caribe, algunas veces muy deformado y afectado por subducción.

\section{Conclusiones}

Las rocas estudiadas, clasificadas como metagabros y afectadas por metamorfismo de bajo grado en facies esquisto verde, conservan la textura ígnea original subofítica, desarrollada entre plagioclasas y piroxenos.

b.

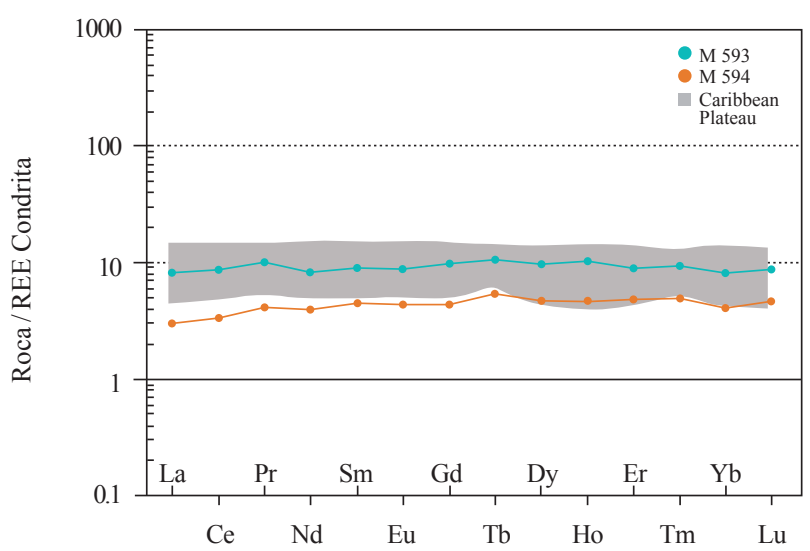

Figura 5. a. Diagrama de REE normalizadas con respecto a la condrita, según Nakamura (1974), el área gris corresponde a el comportamiento típico de rocas de Plateau del Caribe (Kerr et al., 1996). b. Diagrama Nb/Y vs. Zr/Y (Fitton et al., 1997 citado en Thompson et al., 2003), en donde se evidencia que las rocas fueron originadas en plateau del Caribe 
Los análisis geoquímicos y las correlaciones de elementos traza indican que las rocas básicas de la isla Gran Roque poseen un carácter subalcalino y pertenecen a la serie toleítica, con características de rocas de piso oceánico de tipo MORB. Las REE normalizadas con respecto a condrita, manto primitivo y MORB-N, muestran patrones característicos de rocas generadas en dorsales mesooceánicas de tipo normal (MORB-N). Con base en diagramas en los que se muestra el comportamiento típico de rocas generadas en el Plateau del Caribe, se evidenció que las rocas de Gran Roque bajo estudio son muy similares, pues según los análisis realizados tienen características tanto de ambiente MORB como de plateau oceánico.

La presencia de rocas graníticas de arco de islas, que afecta el basamento de gabros y diabasas de la isla de los Roques (Ostos, 1990), es una característica usual en los márgenes de la placa del Caribe y sugiere que el Plateau del Caribe en este sector formó parte del arco volcánico de las Antillas.

\section{Agradecimientos}

Los autores agradecen al Ministerio de Ciencia e Innovación de España, por la financiación del proyecto "Paleobiogeografía del Ordovícico del norte de Sudamérica y sus relaciones con la Cuenca Central Andina y el margen mediterráneo de Gondwana", durante el cual se realizó la visita al área de estudio.

\section{Bibliografía}

Aguerrevere, S., \& López, V. 1938. The geology of the island Gran Roque (Federal Dependencies, Venezuela) and its phosphate deposits. Boletín de Geología y Minería 2: 155-181.

Case, J. E., MacDonald, W. D., \& Fox, P. J. 1990. Caribbean crustal provinces; Seismic and gravity evidence. En Dengo, G., Case, J. E., eds. The Caribbean Region. The Geology of North America, Volume H. Boulder, Colorado, Geological Society of America, p. 15-36.

Diebold, J., \& Driscoll, P. 1999. New insights on the formation of the Caribbean basalt province revealed by multi-channel seismic images of volcanic structures in the Venezuelan Basin. En Mann, P., ed. Caribbean Sedimentary Basins, Sedimentary Basins of the World, Elsevier, p. 561-589.

Donnelly, T.W., Melson, K., Kay, R., Rogers, J.J.W. 1973. Basalts and dolerites of Late Cretaceous age from the central Caribbean. En Edgar, N.T., Saunders, J.B., eds. Initial Reports of the Deep Sea Drilling Project, Volume 15. Washington, DC, United States Goverment, p. 989-1012.

Draper, G., \& Gutiérrez, A. 1997. La estructura del Cinturón de Maimón en la isla de Hispaniola y sus implicaciones geodinámicas. Revista Sociedad Geológica de España, 10: 281-299.

Duncan, R.A. \& Hargraves, R.B. 1984. Plate tectonics evolution of the Caribean region in the mantle reference plane. En
Bonnini, R.B., Hargraves, R.B., eds. The Caribean South America Plate Boundary and Regional Tectonics, Volume 16. GSA Memory, p. 81-93.

Edgar, N.T., Ewing, J.I., \& Hennion, J. 1971. Seismic refraction and reflection in the Caribbean Sea. American Association of Petroleum Geologist Bulletin 55: 833-870.

Fitton, J.G., Saunders, A.D., Norry, M.J., Hardarson, B.S., Taylor, R.N. 1997. Thermal and chemical structure of the Iceland plume. Earth and Planetary Science Letters 153: 197-208.

Gonzáles de Juana, C., Iturralde de Arozena, J.M., \& Picard, X. 1980. Geología de Venezuela y de sus cuencas petrolíferas. Caracas, Foninves, 1031 p.

Irvine, T.N., \& Baragar, W. 1971. A guide to the chemical classification of the common volcanic rock. Canadian Journal of Earth Science 8: 523-548.

James, K.H. 2006. Arguments for and against the Pacific origin of the Caribbean Plate: Discussion, finding for an interAmerican origin. Geologica Acta 4: 279-302.

James, K. H. 2007. The Caribbean Ocean Plateau - an overview, and a different understanding, Caribbean Plateau, Aberystwyth, Wales, UK., University of Wales, Institute of Geography and Earth Science, p. 1-28.

Kerr, A.C., Marriner, G.F., Tarney, J., Nivia, A., Saunders, A.D., Thirlwall, M.F., Sinton, C.W. 1997. Cretaceous basaltic terrains in Western Colombia: Elemental, chronological and $\mathrm{Sr}-\mathrm{Nd}$ isotopic constraints on petrogenesis. Journal of Petrology. 38: 677-702.

Kerr, A.C., Tarney, J., Marriner, G.F., Nivia, A., Klaver, G.T., Saunders, A.D. 1996a. The geochemistry and tectonic setting of late Cretaceous Caribbean and Colombian volcanism. Journal of South America Earth Sciences 9: 111-120.

Kerr, A.C., Tarney, J., Marriner, J.F., Klaver, G.T., Saunders, A.D., Thirlwall, M.F. 1996b. The geochemistry and petrogenesis of the Late-Cretaceous picrites and basalts of Curacao, Netherlands Antilles: A remnant of an oceanic plateau. Contributions to Mineral and Petrology 124: 29-43.

Kerr, A.C., White, R.V., \& Saunders, A.D. 2000. LIP reading: Recognizing oceanic plateaux in the geological record. Journal of Petrology 41: 1041-1056.

Meschede, M. 1986. A method of discriminating between different types of mid-ocean ridge basalts and continental tholeiites with the Nb-Zr-Y diagram. Chemical Geology, 56: 207-218.

Moreno-Sánchez, M., \& Pardo-Trujillo, A. 2003. Stratigraphical and sedimentological constrains on western Colombia: Implications on the evolution of the Caribbean Plate. En Bartolini, C., Buffler, R.T., and Blickwede, J.F., eds. The Circum-Gulf of Mexico and the Caribbean: Hydrocarbon habitats, basin formation, and plate tectonics, American Association of Petroleum Geologist, memoir 79: 891-924.

Nakamura, N. 1974. Determination of REE, Ba, Fe, Mg, Na and K in carbonaceous and ordinary chondrites. Geochimica and Cosmochimica Acta 38: 757-775. 
Nivia, A. 1987. Geochemistry and origin of the Amaime and volcanic sequences Southwestern Colombia [Tesis en Ciencias]: Londres, Universidad de Leicester.

Officer, C. B., Ewing, J. I., Edwards, R. S., \& Johnson, H. R. 1957. Geophysical investigations in the eastern Caribbean, Venezuelan Basin, Antilles Island Arc, and Puerto Rico Trench. GSA Bulletin 68: 359-378.

Ostos, M.R. 1990. Tectonic evolution of the south-central Caribbean based on geochemical data: $\mathrm{Ph}$. D. thesis, Rice University, Houston, Texas, $221 \mathrm{p}$.

Pearce, J.A. 1996. A user's Guide to Basalt Discrimination Diagrams. In Trace Element Geochemistry of Volcanic Rocks: Applications for massive sulphide exploration. Geological association of Canada. Short Course Notes, v. 12.

Pearce, J.A. 2007, Geochemical fingerprinting of oceanic basalts with applications to ophiolite classification and the search for Archean oceanic crust. Lithos 100: 14-48.

Pindell, J., \& Kennan, L. 2001. Kinematic Evolution of the Gulf of Mexico and the Caribbean. Transactions, Petroleum Systems of Deep-water Basins: Global and Gulf of Mexico Experience, GCSSEPM 21st Annual Foundation Bob F. Perkins Research Conference. Houston, Texas, p. 193-220.

Pindell, J.L. 1994. Evolution of the Gulf of Mexico and the Caribbean. En S.K., D., and Jackson, T.A., eds. Caribbean Geology: An introduction. Kingston, Jamaica, University of the West Indies Publishers Association/University of the West Indies Press, p. 13-39.

Santamaría, F., \& Schubert, C. 1974. Geochemistry and geochronology of the Southern Caribbean-Northern Venezuela plate boundary. Bulletin of the Geological Society of America $\mathbf{8 5}$ : 1085-1098.

Saunders, A.D., Tarney, J., Kerr, A.C., \& Kent, R.W. 1996. The formation and fate of large igneous provinces. Lithos 37: 81-95.
Schubert, C., \& Moticska, P. 1972. Reconocimiento Geológico de las Islas Venezolanas en el Mar Caribe entre Los Roques y Los Testigos (Dependencias Federales). Acta Científica Venezolana 23: 210-223.

Sinton, C.W. 1996. A tale of two large igneous provinces: Geochronological and geochemical studies of the North Atlantic volcanic province and the Caribbean oceanic plateau: $\mathrm{Ph}$. D. thesis, Oregon State University, Corvallis, 1993 p.

Sinton, C.W., Duncan, R.A., Storey, M., Lewis, J., Estrada, J.J. 1998. An oceanic flood basalt province within the Caribbean plate. Earth and Planetary Science Letters 155: 221-235.

Sun, S.S., and McDonough, W.F. 1989. Chemical and isotopic systematics of oceanic basalts: Implications for mantle composition and processes. En Saunders, A.D., and Norry, M.J., eds. Magmatism in Ocean Basins, Volume 42: London, Geological Society, Special Publications, p. 313-345.

Thompson, P.M.E., Kempton, P.D., White, R.V., Kerr, A.C., Tarney, J., Saunders, A.D., Fitton, J.G., McBirney, A. 2003. Hf-Nd isotope constraints on the origin of the Cretaceous Caribbean plateau and its relationship to the Galapagos plume. Earth and Planetary Sciences Letters 217: $59-75$.

Urbani, F. 2005. Geología de la isla del Gran Roque, Parque Nacional los Roques, Venezuela: guía de excursión, FUNVISIS y UCV, p. 28.

Wilson, M. 1989. Igneous petrogenesis, a blogal tectonic approach: Dordrecht, Springer, $466 \mathrm{p}$.

Winchester, J.A., \& Floyd, P.A. 1976. Geochemical discrimination of different magma series and their differentiation products using immobile elements. Chemical Geology 20: 325-343. 